\title{
Is the Universe Enough? Can It Suffice as a Basis for Worldviews?
}

Mark Lupisella

Horizons Project

\begin{abstract}
The modern scientific cosmic perspective is unique and compelling, but it is not for everyone. Modern cosmology can be humbling and awe-inspiring, even motivating It can also be overwhelming, even scary. The extent to which the universe we know today can form the basis of satisfactory worldviews rests largely on human psychology, preferences, and needs, as well as on what we mean by "worldview". This essay will explore some ways to think about worldviews and the universe, with an emphasis on exploring relationships between cosmic evolution and cultural evolution (Dick and Lupisella), including what might be called "cosmocultural evolution" - the coevolution of cosmos and culture (Lupisella 2009). We will touch on a few cosmocultural evolutionary perspectives as well as broader underlying "cosmological theories of value". With an eye toward psychology, we will consider if and how such perspectives might inform, or possibly suffice as worldviews, suggesting generally that the universe may suffice for some people some of the time, but probably not for most people most of the time.
\end{abstract}

Correspondence | Mark Lupisella, Mark@HorizonsProject.org

Citation | Lupisella, M. (2019) Is the Universe Enough? Can It Suffice as a Basis for Worldviews? Journal of Big History, III(3); 123 - 140.

DOI | https://doi.org/10.22339/jbh.v3i3.3370

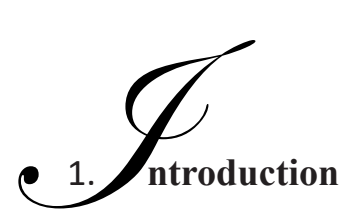

"The Cosmos is all that is, all that was, and all that ever will be."

- Carl Sagan ${ }^{1}$

"If the universe isn't enough, what are we to do?"

- Ian Crawford ${ }^{2}$

The modern scientific cosmic perspective is unique and compelling. But it is not for everyone. It may only be compelling to a small fraction of people. Modern scientific cosmology can be humbling and awe-inspiring, even motivating to some. But it can also be overwhelming and even scary. The extent to which the universe we know today can form the basis

1 Sagan, C. 1980. Cosmos. New York: Random House. P 4.

2 Personal email, 24 April 2018. of satisfactory worldviews rests largely on human psychology, preferences, and needs, as well as on what we mean by "worldview".

When I first learned of the Australian National University Symposium on "Expanding Worldviews: Astrobiology, Big History, and the Social and Intellectual Benefits of the Cosmic Perspective" (summarised by Crawford 2018), I was immediately struck by the explicit reference in the title to "social benefits of the cosmic perspective." Much effort has gone into our modern understanding of the universe, but there appears to be a relative lack of attention given to the question of how such pursuits truly benefit society (Race et al 2012, Dick 2018). ${ }^{3}$ Maybe that is because the benefit is obvious enough since much of

3 A NASA workshop on the societal implications of astrobiology was held in 1999 (NASA Technical Memorandum 1999), and there have been subsequent efforts to explicitly explore the connections between astrobiology and society (e.g., Race et al 2012 and Dick 2018). 
the human population seems to deeply value learning about our world - including learning about our broader universe even though it is extremely old and vast and largely detached from everyday human life.

Indeed, even with some perceived detachment of human life from the larger universe, many societies still seem to be willing to conduct extensive exploration of our cosmos even though it does not have much, if any, operational impact on our daily lives. This says something about the human species - many of us are compelled to learn about almost anything, and society provides resources to engage in what are often timeconsuming, life-long, multi-generational, expensive endeavours to learn about things we suspect will not have near-term practical relevance for our lives.

We like to learn because we are interested. We are presumably interested for at least evolutionary reasons since there have likely been strong selective pressures to be curious about our wider world, which would lead to a better understanding for practical living, which can then lead to better individual and group fitness. But many modern humans, who arguably now have more time and cognitive processing power to pursue "impractical" questions, are also intrigued by the often vexing philosophical questions regarding value, meaning, and purpose, and why we observe the universe we do, or why the universe exists at all.

I am personally charmed and awed by our universe (even more so by the possibility of a multiverse!), and by wondering if it is "about something" and why it exists in the first place. I have spent a good amount of mental energy on such things (perhaps an irrational amount), but my fascination and intrigue is not necessarily widely shared by others - some of who presumably think they already have answers in forms of religions or other philosophies and worldviews that have been around for thousands of years. And my interest arguably has something to do with personal psychological predispositions that do not benefit others that much, if at all. But given the importance of worldviews and the bewildering variety that complicates our ability to know what is true, and given the sometimes deeply problematic adverse affects that can result from many worldviews, it does seem worth asking whether the scientific universe we know today, or may know in the future, can at least help inform and/or form the basis of satisfactory worldviews.

It would seem that any hope for building satisfying cosmological worldviews would need to entertain some degree of integration, if not full integration, between physical cosmic evolution and the emerging meaningful powerful cultural evolution occurring here on earth and perhaps elsewhere in the universe. Fortunately, our modern scientific understanding of cosmology provides a significant amount of that integration. Modern cosmology tells us that stars, planets, life and humanity are the results of a long process of micro-scale and large-scale cosmic evolution, including biological and cultural evolution - at least in our little corner of the cosmos. "The Cosmos is within us. We are made of star stuff." - as Carl Sagan famously proclaimed. ${ }^{4}$

Taking a cue from our emerging integrated scientific story of the universe, this paper will explore a few ways to think about worldviews and the universe, with an emphasis on exploring relationships between cosmic evolution and cultural evolution, including what might be called "cosmocultural evolution" - the coevolution of cosmos and culture (Lupisella 2009). We will touch on a few cosmocultural evolutionary perspectives as well as broader underlying "cosmological theories of value" being developed for an upcoming book, Cosmological Theories of Value: Science, Philosophy, and Meaning in Cosmic Evolution. We will address if and how cosmic perspectives might inform, or provide a basis for, alternative "cosmological worldviews."

4 Sagan, C. 1980. Cosmos. Random House. P. 244. 
This short treatment cannot include the myriad details of cosmic evolution and all the relevant details regarding the philosophy and psychology of belief and worldviews and how they impact human behavior, but we will certainly draw from some of that work, much of which can be found in academic and popular treatments, e.g.: Aerts et al 1994 and 1999, Babbage and Ronan 2000, Wilson 2002, Shermer 2002, Koltko-Rivera 2004, Gershenson et al 2007, Bulbulia et al 2008, Johnson et al 2011, Henriques 2011, Vidal 2012, Nilsson 2013, 2014, 2015, Hedlund de-Witt et al 2014, Saucier 2013, 2015).

\section{The Universe and Worldviews}

\subsection{Why Care About Worldviews?}

\section{"A belief is a lever that, once pulled, moves almost everything in a person's life."}

- Sam Harris (2005, p 12)

Beliefs and worldviews are different from each other, but they are usually intimately related. Many, but not all worldviews can include beliefs that are not necessarily grounded in much evidence or careful investigation or reasoning. But worldviews can also be evidence-driven. They can be highly complex and diverse, but there tend to be some common underlying drivers, motivations, and themes associated with the psychology and content of many worldviews that can be used to help assess the extent to which our universe can serve as, or at least inform potentially satisfying worldviews.

We can start with a relatively simple definition of 'worldview', which is to define it essentially like it sounds: a view of the world. 'View' often implies particular perspectives and beliefs. 'World' often implies everything (or almost everything). A worldview, then, is a kind of "view of everything" that may matter in a person's life or a group's functioning, e.g., survival, human affairs, facts and values, meaning and purpose, death and afterlife, epistemology and ontology, transcendent realities, etc. This is not different in its essence from characterizations offered by others, and given this kind of characterization, we can see why worldviews can drive very specific details of what we believe, how we think, and why we act in certain ways (Koltko-Rivera 2004, Johnson et al 2011, Vidal 2012). Worldviews can be comforting and inspiring, but also dangerous. ${ }^{5}$

Strictly scientific narratives and worldviews, including those based on our present state of physical cosmology, can be limited or even misguided, and perhaps dangerous as well, so we should be mindful of potential pitfalls, including what might be called "oppressive universalism" 6 or "overfoundationalizing" (Rockmore and Singer 1992). These are not just legitimate psychological concerns about the misuse of worldviews, but they are also legitimate intellectual concerns that are particularly important when engaging in speculative worldview building - especially when that worldview building is driven by contemplations of our entire universe and the associated complexities of modern cosmology. Keeping these sensitivities in mind (Denning 2009), we can explore "cosmological worldviews", which can be thought of as worldviews that are heavily informed by cosmology, i.e. by modern scientific cosmic evolution that includes fundamental physics such as relativity and quantum mechanics (that drives much cosmic evolution), as well as biological evolution, including the evolution of intelligence and culture.

5 Juergensmeyer (2003) looks closely at the links between violence and a number of religions, but its important to note that while worldviews are often associated with religions and theology, they are not limited to those orientations.

6 I use the phrase "oppressive universalism" here as a way to capture to the idea that "universe narratives" can be misguided and oppressive, including to the extent that they may deemphasize individualism in favor of very broad narratives (Marshall 2002). 


\subsection{Theology}

The universe and worldviews have been intimately connected for thousands of years. Ancient and modern religions have found many ways to integrate concepts of the larger universe into their worldviews. Western religions such as Judaism, Christianity, and Islam have tended to emphasize the universe as God's creation. Hinduism has proffered notions of a very long-lived, if not eternal, cyclical universe. Pantheism has generally equated the universe with divinity or "God", and panentheism has viewed the universe as imbued with divine spirit that also transcends the universe. More generally and more recently, some have referred to "cosmotheology" as an attempt to capture the idea that notions of spirit or divinity or God should tightly integrate - if not be fully constituted by details of modern scientific cosmic evolution (Dick 2000). ${ }^{7}$ Some eschatological treatments have tended to emphasize "end-points" of cosmic evolution, for example, leading to a super advanced intelligent "Godlike" being, or state, at the end of cosmic evolution (e.g. Teilhard de Chardin 1955, Tipler 1994).

\subsection{Speculative Cosmology}

There are numerous scientific treatments, or what could perhaps be thought of as more "secular" speculative philosophical treatments, that have potential relevance for the universe and worldview building - at least by way of informing alternative worldviews, if not having the potential to fully constitute worldviews in and of themselves. There have been articulations of cosmic evolution that emphasize a kind of "spiritual" embrace of our universe without necessarily explicitly emphasizing theological or divine dimensions or heavy philosophical treatments that explicitly invoke metaphysics or value theory (e.g.

7 Kant appears to have coined the term "cosmotheology" in Critique of Pure Reason to capture the idea that a "supreme being" might be inferred by experience of the world. Steve Dick's (2002) more contemporary use is different in that it does not require a "supreme being".
Swimme and Berry 1992, Barlow 1997, Goodenough 1998). "Big History" treatments emphasize a cosmicscale view of history and some level of comprehensive integration that includes the evolution of life and humanity (Christian 2004).

Anthropic views emphasize the idea that our observed universe appears as it does because is it consistent with the evolution of beings that can eventually observe it. Multiverse concepts posit the existence of many, possibly infinite, universes and is often used to explain our particular cosmic details (e.g. laws and constants) by noting that the existence of many other universes makes our particular universe less improbable than it may otherwise appear to be. Cosmological Natural Selection suggests that as universes give "birth" to other universes (possibly via black hole production), a kind of selection process would tend to produce relatively stable and long-lived universes such as ours (Smolin 1997).

Information-based views of the universe have been proposed noting that the universe can be seen as a kind of computational system (Lloyd 2006). Ideas such as the "evolutionary developmental universe" (Smart 2009), taking cues from biological evolution, emphasize how the evolution of intelligent beings can lead to highly computational systems such as a "developmental singularity", perhaps in the form of a "black hole computing system" that can give rise to similar universes with incremental changes. Related to ideas of an information-based universe, it has been suggested that our universe may actually be a simulation (Bostrom 2003).

James Gardner (2005), leveraging ideas from John Wheeler who suggested some degree of "retrocausation" might be possible, proposes a kind of participatory or "co-created" evolutionary model of the universe as a "closed time-like curve", which can provide a theoretical explanation for a "selfsynthesized" origin and evolution of the cosmos. 
Paul Davies (2009) goes further, speculating that the universe and its specific bio-friendly laws might be "self-synthesizing" via cultural evolution leading to cosmic-scale "retroactive" observer-participancy in which the whole universe, eventually "saturated by mind", essentially "retro-actively" brings itself and its specific laws into being (at least by constraining "past" possibilities, in which case it can be thought of "retro-constraining").

\subsection{Cosmophilosophy}

For lack of a better phrase, I would like to also add "cosmophilosophy" as a category that overlaps with much of what has been touched on prior, but adds a more explicit and systemic treatment on relationships between contemporary cosmology (scientific cosmic evolution) and an explicit emphasis on philosophical questions of value, meaning, agency, epistemology, and metaphysics. Cosmophilosophy asks, in part, questions having to do with what value might be associated with the universe and its evolution, whether there is any meaning or purpose in the cosmos, why it has evolved in the way we think it has, or why the universe exists at all. Here, we will very briefly touch on three "cosmological theories of value" (cosmological reverence, cosmocultural evolution, and the connection-action principle) taken from a past book chapter (Lupisella 2016) and an upcoming book (Lupisella in-press). In the next section we will assess to what extent these theories of value and other related ideas touched on previously might provide a basis for worldviews.

\section{Cosmological Reverence}

Similar to what was noted in the speculative cosmology section above (without the stronger "spiritual" invocations), cosmological reverence can be seen a sub-category of cosmophilosophy that captures ideas suggesting we can deeply revere the universe for a variety of reasons, including that we are intimately related to, and dependent on the universe since we arose from a long complex process of cosmic evolution and rely on the universe's material and energy for our existence and future evolution. We can revere the universe for purely scientific reasons, as well as any awe and majesty we might have in the face of the universe's magnitude, mysteries and complex evolution (Carroll 2016). The definition of cosmological reverence suggested here is a kind of one-way relationship in the sense that it is limited to the cosmos being significant for us, but not the reverse. Cosmological reverence recognizes that we are a product of, and sustained by the universe, but does not claim that we have any particular significance for the universe at large.

\section{Cosmocultural Evolution}

Cosmocultural Evolution stresses the idea that physical cosmic evolution and emerging cultural evolution are co-evolving and will continue to more tightly co-evolve in the future, with both having significance for each other - both are evolutionary dynamics that are in some sense on par with each other in terms of significance. One way to think about culture is as the "collective manifestation of value" - where value is that which is valuable to "sufficiently complex" agents, from which meaning, purpose, ethics, and aesthetics can be derived. Culture manifests value in many varied forms, from thoughts and knowledge to symbolic abstractions to social norms and organizations to mass movements and large-scale creations (Lupisella 2009).

We should avoid such a strong distinction between cosmic evolution and cultural evolution that they are thought of as completely distinct from each other. Cultural evolution is ultimately a part of cosmic evolution in the broad sense that culture has emerged as part of the physical evolution of the universe. However, we can make a useful distinction to the extent that culture is a different enough evolutionary phenomenon from the rest of physical cosmic 
evolution. It can be a useful distinction to the extent that it can help address the interesting question of how significant cultural evolution may be in a cosmic context.

One version of a cosmocultural evolution perspective can be thought of as "bootstrapped cosmocultural evolution" which suggests the universe has "bootstrapped" itself into the realms of value, meaning, and purpose via culturally evolving beings like ourselves - but for no particular reason other than the physical characteristics of the universe allowed for life and intelligence to emerge and evolve naturally. Stronger versions suggests that cultural evolution could become a very significant, if not dominant form of evolution with possibly infinite potential and significance - similar to ideas touched on previously. Cosmocultural evolution suggests that cultural beings may become, and perhaps already are, a kind of cerebral cortex for the universe - a source of self-awareness and intentional creators and arbiters of value, meaning, and purpose (Lupisella 2009). It seems we are a way for the universe to not only know itself, but to value itself.

\section{Connection-Action Principle}

Treading deeper into the stormy waters of what is arguably at least part metaphysics, we can ask why the universe exists at all and why its evolution appears to have been a very long-lived, highly dynamic and creative process. What is its "source", if any? A brutefact explanation would generally refer to the laws of physics and/or initial conditions of the universe as facts to accept without cause or explanation, and those laws and conditions explain why and how the universe evolves and creates. More specifically, a purely scientific explanation would suggest that an initial high-energy, low entropy state naturally gave rise to expansion, ${ }^{8}$ with cooling and "clumping"

8 The expansion may have included an extremely rapid and unusually accelerated "inflationary" expansion suggested by inflationary theory (Guth and Steinhardt 1984, Linde 1994, emerging over time, consistent with the second law of thermodynamics, gravity, and other physical forces, causing the aggregation or "creation" of objects like atoms, stars, galaxies and planets.

But we can still ask: why those initial conditions? Why these laws? Even more challenging, why any "order" or laws to begin with? Why an origin at all, and was it truly from "nothing" as some have suggested?" Merely being able to ask these questions does not mean they are well-posed or have answers, let alone scientific answers, but there have been many suggestions, some of which overlap heavily with what was touched on prior, including, for example: (1) design by a God or gods or some kind of entity or beings, including the possibility that our universe is a simulation of sorts (Bostrom 2003), (2) anthropic principles (e.g. Barrow and Tipler 1986), (3) an eternally oscillating universe, going back to the Greeks and forms of eastern worldviews such as Hinduism and now by some in modern cosmology (e.g. Steinhardt and Turok 2002), (4) cosmological natural selection (Smolin 1997), (5) a metaverse or multiverse that suggests the possibility of many universes (Tegmark 2003), to (6) even more provocative versions of anthropocentric thinking that suggest conscious beings in some sense create the universe and possibly even its laws via extreme interpretations of quantum mechanics - as touched on previously (von Neumann 1932, Wheeler 1990, Davies 2009).

Regardless of the kind of explanation for our universe's origin and its particular laws and initial conditions, most suggestions seem to rest on, assume, or at least imply that our universe is dynamic. It appears we live in a universe of action - and action is central to our understanding of our universe (Turchin 1993,

Guth 1998)

9 See Krauss (2012) for a recent scientific exploration of an origin from nothing, but which nevertheless seems to fall short of explaining the emergence from truly "nothing" - at least in the traditional philosophical sense of truly nothing (itself a premise Krauss appears to challenge). 
Mermin 2017). ${ }^{10}$ Even contemplations of an origin as a quantum fluctuation from a quantum vacuum state or quantum "foam" (a realm of virtual particles which are wavelike fluctuations in the quantum vacuum at "absolute zero") seems to imply there is still "something" that is "dynamic". The quantum vacuum state appears to at least posit, if not be in actuality, a realm of action, or at least a realm that gives rise to some form action - as if the quantum vacuum state itself is unstable and must produce action. We seem to live in a fundamentally action-laden universe. But why should there be any action all?

The connection-action principle (CAP), in its simplest form, makes the conceptual suggestion that the universe's property of connectedness is manifested as action - perhaps in ever-increasing degrees and perhaps necessarily so in stronger versions that might be something more like: the universe's necessary property of connectedness is necessarily instantiated as relations and actions and increasing degrees thereof (Lupisella 2016, Lupisella 2019 forthcoming) as indicated by the simple graphic below (Figure 1).

This admittedly speculative suggestion leverages

10 Valentin Turchin (1993) explicitly, and seemingly necessarily, links the epistemological criticality of action with an action ontology. Mermin (2017, p 89) emphasizes the importance of action when he writes of QBism: "in QBism, on the other hand, a measurement can be any action taken by any user

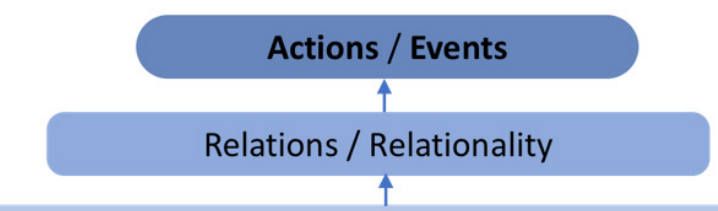

Universal Property of Connectedness

Figure 1: Graphical Representation of the Connection-Action Principle

on her external world. The outcome of the measurement is the experience the world induces back in that particular user, through its response to her action." This is very similar to Relational Quantum Mechanics which is touched on subsequently. the old idea of the connectedness of the universe (Sciama 1959, Bohm and Hiley 1993), but goes further and suggests that for the property of connectedness to be realized, something needs to happen, some action or event needs to instantiate and realize any connectedness or relationship, where relations can be thought of as slightly more specific and more concrete forms of connectedness. In that conceptual sense, the property of connectedness is a source of action, a kind of "cause" for action. An action occurs to help realize connectedness and relations, i.e. to make connectedness and relationality real or actual, to manifest and instantiate what can be thought of as a kind of "relational potential" of the universe.

The connection-action principle is arguably consistent with relational metaphysics in general and process philosophy more specifically (for which Whitehead's "actual events" are a critical element of his ontology (Whitehead 1929) and may provide a conceptual explanation for why there should be relations, processes, actions, and events at all. CAP is arguably consistent with a number of ideas such as Action Ontology (Turchin 1993) and other ideas such as Relational Quantum Mechanics (Rovelli 1996), quantum entanglement (potentially revealing an additional form of deep connectedness), and information-based ontologies (Lloyd 2006, Davies and Gregersen 2010), including Bohm's notion of "active information" (Bohm and Hiley 1984, 1987, 1993; Bohm 1989) ${ }^{11}$ - where information can be thought of as the details that characterize and specify relations.

Even quantum field theory (QFT), an increasingly prevalent and successful practical framework for quantum mechanics which leverages field constructs, can also be viewed as suggesting deep degrees of connectedness and relationality in the sense that

11 There are a number of speculative and far-reaching applications of active information to psychology and mind, including connections to value and meaning (e.g. Pickering 1995, Pylkkänen 2016). 
the notion of a field is a singular seamless "intraconnected" construct that fundamentally drives and manifests physical dynamics. Notably, the field construct can arguably be traced back to Newton who speculated about some "action at a distance" between bodies to help explain gravitational forces. QFT can also be traced more recently to a third major early formulation of quantum theory (the first two coming from Schrodinger and Heisenberg) which was first developed by Paul Dirac and has been called, notably, the Interaction Picture (Sakurai and Napolitano 2017) - arguably consistent with some interpretations of the Connection-Action Principle.

A stronger version of the connection action principle suggests that the universe increasingly manifests its property of connectedness through increasing degrees of action (and hence increasing degrees of diversity and complexity, etc.). The connection action principle is arguably consistent with (a) Many Worlds interpretations of quantum mechanics, (b) the multiverse concept, and (c) the temporal version of the Principle of Plenitude (Lovejoy 1936) in the sense that they can all be seen as examples of robust realizations of CAP because they produce increasing degrees of action in the form of complexity, extreme diversity, and creativity more generally.

This kind of theoretical conceptual proposal can be interpreted to suggest that the universe is "about something" - something admittedly general and perhaps highly open-ended and even vague, but if the universe is about something like realizing connectedness through action, we can further interpret that to suggest there is a kind of value associated with the universe's action-laden evolution - a value "intrinsic" to the nature of universe. In this view, cosmic evolution can be generally seen as a realization of the universe's potential, and specifically, the more "action" in the universe (where, again, action can be interpreted very broadly, including creating new relations, "objects", complexity, diversity, etc.), the more its nature is realized. This can then lead us to ask if and how this kind of speculative metaphysics might directly or indirectly inform worldviews, values, meaning, purpose, etc.

\section{Can The Universe Be Enough?}

Here we will consider a slightly different question from the title and ask: can the universe be enough? This will allow for a more general, theoretical, and future-oriented assessment. Whether the universe can be enough to provide a sufficient basis for worldviews depends on many details - including details of the worldview itself and the needs of the individuals and groups holding the worldview(s). There are many ways we can go about addressing questions regarding if and how the universe can suffice as a basis for worldviews. We will draw from a few sources to develop some lenses through which to assess the utility of the cosmological worldview ideas touched on prior, with an emphasis on the cosmophilosophy ideas that attempt to explicitly address philosophical questions of value, meaning, and purpose in the context of modern cosmology.

\subsection{Assessment Methods}

We can start by considering three general lenses through which to analyze the worldviews touched on in this essay: science, psychology, and philosophy - all of which overlap and inform each other as indicated in Figure 2. Clement Vidal (2012) offers a comprehensive and detailed framework for evaluating worldviews, particularly in the context of modern cosmology (Vidal 2014), consisting of: (A) 6 philosophical dimensions (descriptive, normative, practical, critical, dialectical, synthetic), (B) a philosophical agenda for defining what a worldview is, (C) several "objective", "subjective", and "inter-subjective" evaluation criteria, and (D) a set of tests, including "first order" tests of is-ought, ought-act, and is-act. This paper will loosely apply the evaluation criteria from item $\mathrm{C}$ above: objective criteria of internal consistency, consistency with 
science, broad scope; subjective criteria of utility, consistency with personal experience, emotional value; and inter-subjective criteria that address social factors such as collective utility, reducing conflict, and communicability.

Interestingly, as suggested in Figure 2, while not a perfect mapping, Vidal's worldview evaluation criteria categories (objective, subjective and intersubjective) map reasonably well to the categories of science, psychology and philosophy in the sense that science attempts to pursue objectivity, psychology is more about subjective personal experience (with increasingly powerful scientific methodologies), and philosophy has a lot to do with how people think, value and act with respect to each other (also subject to scientific investigation, e.g. including via social psychology). The philosophy lens we will use here may differ from Vidal's inter-subjective category in the sense that his inter-subjective category is arguably

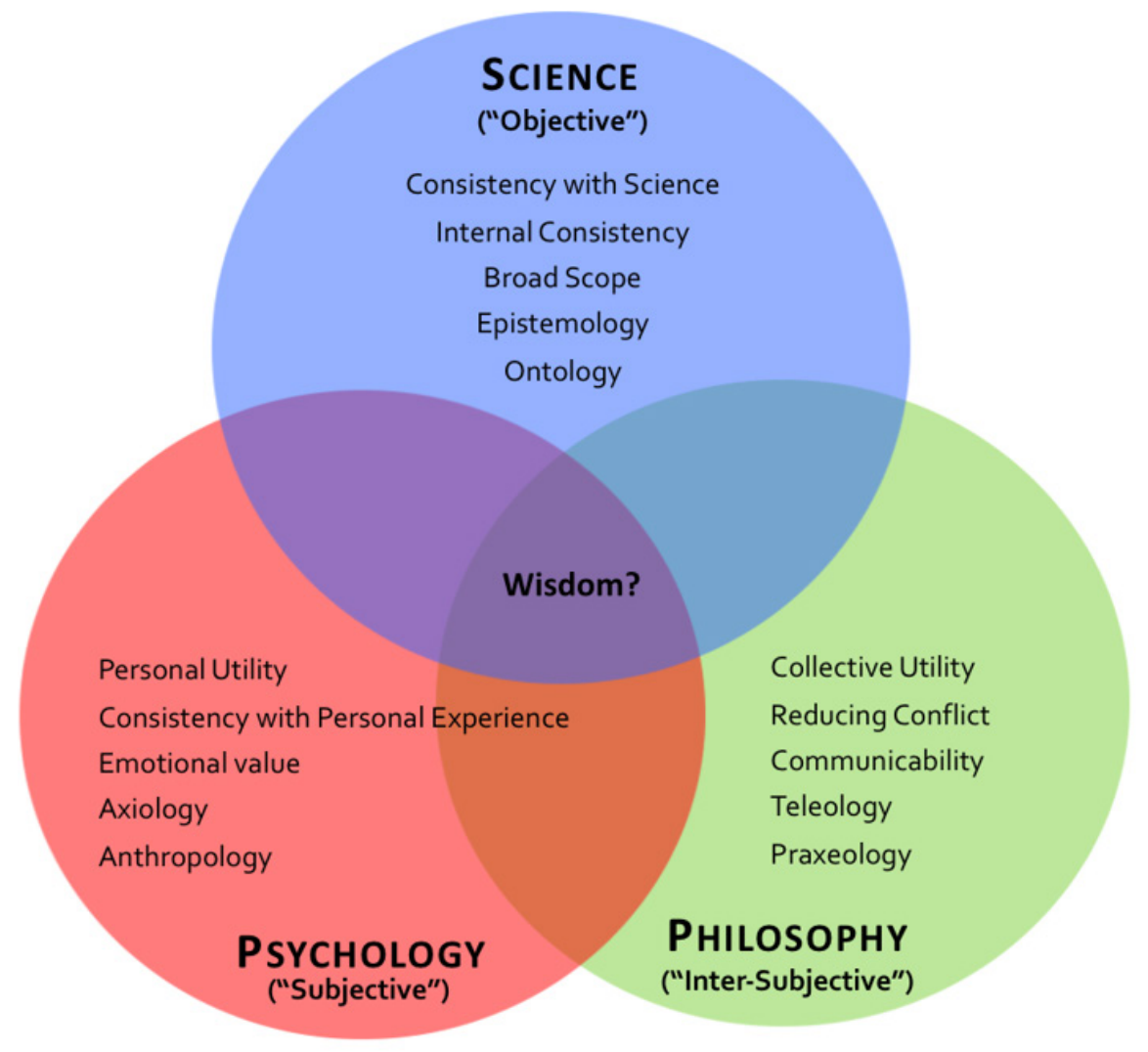

"Figure 2: Three Worldview Evaluation Lenses" a bit more pragmatic for group functioning, while the philosophy lens I have in mind is more aspirational, speculative, and theoretical (but with potentially important practical consequences). So, while there is critical overlap between these three lenses, ${ }^{12}$ to simplify the usage in this essay, we might summarize by saying that science attempts to understand what is real about the world, psychology is more about how people actually operate, and philosophy is more aspirational and speculative.

While Vidal's framework is closest to the 3 kinds of lenses I would like to use for this essay, Figure 2 also includes key elements from other frameworks for how to think about worldviews. Johnson et all (2011) suggest 6 general areas for worldviews that interrelate and can help integrate between culture and religion. I list 5 of those in Figure 1: ontology, epistemology, axiology (proximate goals and values), teleology (ultimate goals), and praxeology (codes of behavior). Hedlund-deWitt (2012) builds on previous work and constructs a 5-part "integrated worldview framework", two of which are "anthropology" and "social vision" (the other 3 are ontology, epistemology, axiology - similar to Johnson et al). "Anthropology" is described as a perspective on who and what the human being is and any potential roles and positions we might have in the universe. This articulation is helpful for the purposes of this essay and is arguably different enough from other factors to list explicitly. ${ }^{13}$ Hedlund-de-Witt's $12 \quad$ Psychology is obviously informed by science, empirical study, etc., including via emerging fields such as evolutionary psychology. Philosophical, speculative, normative explorations should be informed by science, but not limited by science.

13 Hedlund-de-Witt's "anthropology" is also related to teleology and other areas shown under science and philosophy, but 
"social vision" is arguably captured by other elements listed such as collective utility and praxeology. ${ }^{14}$

Worldview evaluations would likely come out differently depending on any number of factors regarding how we think about the categories and criteria, including how they are weighted, but we can keep this overall kind of evaluation framework in mind as we briefly assess if and how the universe might suffice as a basis for worldviews. It is notable for our assessment that Vidal suggests twice as many subjective criteria than objective criteria, which is important for evaluating worldviews since they often need to address a range of complex subjective human needs and interests.

\subsection{Cosmological Worldview Assessments}

Most of the cosmological worldviews noted previously are arguably strong on the scientific/ objective criteria (e.g. internal consistency, consistency with science, broad scope, etc.) with the exception that depending on the interpretation of traditional theological views (e.g. whether modern science is significantly incorporated), those views can be seen as weaker on the objective and scientific criteria. Indeed the point of most of the cosmological worldviews noted here is to be more consistent with modern science and modern cosmology specifically. However, most of the views noted prior are arguably relatively weak on most of the subjective psychological and philosophical criteria, much of which are presumably driven by natural selection and evolutionary psychology, including group selection -

since it is somewhat narrow in the sense of having an important individualistic component and being limited to anthropocentric views, I have included it in the psychology lens.

14 Obasi (2002) develops a 41 item "Worldview Analysis Scale" targeted primarily for people of African and European descent. Project Worldview is an online collection of many different facets of worldviews and provides diverse guidance for thinking about and analyzing worldviews: http://www. projectworldview.org/welcome.htm. e.g. coping with uncertainty and death, maintaining group cohesion and efficacy, etc. (Wilson 2002, Haidt 2012).

Cosmological Reverence suggests we can value the universe because we emerged from it and are intimately bound up in it, but we do not have any particular significance for the universe at large. So while there can be some emotional value as well as personal and collective utility (including communicability), presumably the emotional value would be limited, not just because we are not significant for the universe and cannot discern important future-oriented implications, but also in part because it is arguably difficult for many people to personally or collectively identify with our immensely old, large, and seemingly impersonal and indifferent universe. More specifically, such a view does not have much, if any, specific practical consequence or utility for dealing with social challenges such as reducing conflict - with the possible exception that revering the universe can help us revere each other as products of cosmic evolution and hence deal with each other more respectfully. Cosmological reverence is primarily a kind of one-way "passive reverence" for the cosmos, but it can nevertheless inspire and inform certain ethical views such as how we might value certain cosmic creations, including each other and other life-forms more generally (Lupisella 2013).

Cosmocultural Evolution is a stronger view in the sense that it can suggest a certain amount of responsibility (perhaps a kind of "cosmic" responsibility?) for intelligent beings since cultural evolution has the potential to have much, perhaps unlimited, significance for the cosmos - but again, for no other reason other than cultural agents arose via physical processes and now have agency and can choose and act on forms of cosmocultural evolution value systems or worldviews. On this view, we can see not only the kind of significance noted by Paul Davies and others that "Somehow, the universe has engineered not only its own self-awareness, but its own self-comprehension" (Davies 2009, 385), but 
also, as noted prior, that the universe has "engineered" its own self-valuing. This might have some emotional value in the sense that it can be seen to provide compelling cosmic significance specifically for beings like ourselves. We may see ourselves as a source of cosmic value where there otherwise may have been none prior. Such a view might have more social intersubjective philosophical value in that it can provide groups of people, or perhaps all intelligent/cultural beings in general, with a common/collective sense of meaning and purpose within what may be the largest shared context possible - the universe.

The Connection-Action Principle goes much further and can be interpreted to imply value based on a claim about the nature of the universe. As touched on prior, while the suggestion is arguably supported by a number of lines of philosophical reasoning and has some consistency with scientific and philosophical ideas, it is nevertheless essentially speculative metaphysics that arguably lacks sufficient physical commensurate evidence or sufficient predictions and tests needed to be persuasive and adopted as a convincing worldview. Its value-based implications are not likely to be something many people could easily identify with or defend. Manifesting the connectedness of the universe through myriad forms of relations and actions may have some appeal and moderate practical consequence in the sense that our connections with others and our wider world might motivate us to act on behalf of those connections and relationships, but details beyond that may not ring true for many people given the highly speculative, conceptual, and abstract nature of the claims. ${ }^{15}$

Cosmocultural evolution, particularly bootstrapped cosmocultural evolution, seems like it might be a tenable "meaningful" cosmological worldview for beings like ourselves because while it may seem

15 We should also be sensitive to concerns that in the worst case, tying a speculative form metaphysics to human affairs can be dangerously problematic depending on how certain details are developed and used. somewhat speculative, it does appear to be defensible to say that the universe has essentially "bootstrapped" itself into the realms of value, meaning, and purpose at least in the form of human minds, if not in others as well. This realm of value, meaning, and purpose has then emerged in the universe through cosmic evolution, through the evolution and emergence of our minds and perhaps other minds that may exist throughout the universe. And the potential for this valuing capacity, for the meaning-making and purpose-seeking we do with our cultural evolution, may have unlimited potential for the universe as a whole. We may be a way for the universe to value itself and find many different, perhaps infinite, evolutionary paths forward.

There may be other forms of value independent of beings like ourselves, but it does nevertheless appear that beings like us are at least one means by which the universe is finding or "discovering" forms of value, meaning, and purpose in what may be an extremely large, if not infinite, possibility space of those qualities. If there is no broader objective meaning and purpose in the universe beyond that which is created by cultural beings, then that realization may help us value each other more.

The claim that value, meaning, and purpose have emerged in the universe as a product of cosmic evolution is in some sense a minimalist view (some may say it is trivially true), but it is potentially significant nonetheless. It is intellectually and philosophically minimalist in the sense there is no need to invoke some other kind of dynamic or force or substance in the world such as spirit or God. There is no appeal to a wholly other "transcendent" reality. However, the implications and significance are still notable in the sense that if value, meaning, and purpose has emerged in the universe through us, then we are arguably "responsible" for it. We are creators and arbiters of value that not only makes the universe valuable, but we also pursue very specific forms of value, e.g. having to do with morality and 
ethics and endless forms of creativity. Indeed, if we choose, we can make the universe "purposeful" in the sense of enabling trends and choosing "directions" for the universe. It is up to us to decide, to choose. Presumably there will be many such diverse pursuits which call for careful deliberation and pluralistic meaning-making with each other.

\section{Synthesis and Summary}

If our worldviews need to be comprehensive and include specific guidance for human behavior and address most of our complex subjective needs, then the universe is probably not enough for most people most of the time - more would be needed to help address, and perhaps compel, certain kinds of human expectations and behavior. Also, there are broader questions such as why the universe exists at all, or more generally, why there is something rather than nothing, that modern cosmology arguably does not provide satisfying answers for - and "brute fact" scientific explanations often don't suffice for many - partly because they do not personally resonate for most people.

From a more philosophical perspective, even if the universe is "about something", if there is some fundamental cosmic nature to be realized (e.g. as suggested by the connect-action principle, which in theory allows us to "derive" "intrinsic" value from something we think the universe may be about) it is still arguably too non-specific and abstract for most people to identify with. Further, it is not clear that intelligent beings must adhere to, or adopt, pursuits consistent with what think our universe is about. We may of course be wrong about what we think the universe is about, and even if we are right about the "facts", the science, or whatever metaphysics is relevant, the old philosophical fact-value or "isought" distinction (including the "naturalistic fallacy" (Moore 1903)) still arguably gives us an option to freely pursue aspirations beyond our understanding of how the world is.
Nevertheless, for some people some of time (possibly all the time for some people), the universe could suffice as a basis for a worldview depending on certain details of the worldview and the needs of the individuals and groups. If a person or group can sufficiently identify with the universe then the universe might be a sufficient overall worldview construct particularly if some value or meaning, however loose and high-level it might be, can be inferred from cosmic evolution (e.g. forms of cosmocultural evolution). Those who do not need a worldview with many, or any, prescriptive details for guiding human behavior, might also see the universe as a sufficient basis for a worldview to the extent that they do not need it to bridge into details of human life - e.g. to provide some sense of caring or how to deal with death, etc.

We might infer from some of the above reasoning that any "single" worldview might not be able to address the full breadth of human needs that many individuals or groups have. The universe can be a big part of a worldview or be one of a few simultaneously operating worldviews (inter-related or not). We can revere the universe, and maybe even see ourselves as integral to its evolution (e.g. cosmocultural evolution), but how we choose to guide our human actions can be independent of any broader cosmological worldview. We can have a kind of hybrid worldview, or a 2-part worldview - one for the universe and one for the details of human life - for which there can be important overlap and relationships, but for which neither completely informs, determines, or depends on the other. As touched on prior, our broader scientific knowledge about the universe can lead us to see ourselves as having randomly evolved from cosmic evolution without any larger cosmic purpose, but with a potential implication that we can see ourselves as needing each other to make our way in an otherwise indifferent universe. ${ }^{16}$

16 Secular humanism is arguably a minimalist science-based worldview that informs human ethics and can be added to more explicit cosmological perspectives that provide a broader sense of reverence and meaning beyond secular 
We can also be sensitive to the idea that we might be asking too much of our worldviews if we expect them to provide answers to everything. We should be mindful of the possibility that no combination of worldviews would necessarily provide complete and irrefutable answers or satisfying sources of comfort, meaning, and purpose for all of our questions and needs. We may be misguided, or at least unsatisfied - and possibly deeply disappointed and adversely psychologically affected - if we expect our worldviews to provide too much. Living with uncertainty is challenging for many, but we do it. Indeed, there appear to be many people who do not require worldviews that provide answers to everything. Those who claim they are "unaffiliated" with any religion make up the third largest group in the world - about 16\% as of 2015 (Pew Research Center 2017). This does not mean the unaffiliated do not have any theistic, deistic, or spiritual beliefs, but it does arguably imply that a large number of people do not need traditional "comprehensive" religious worldviews that prescribe details for human living and answers to many other questions. However, many of those who are unaffiliated with religion almost certainly have some kind of worldview(s). ${ }^{17}$

So for now, a reasonable conjecture as to whether the universe can be enough to suffice as a basis for worldviews is that while it might suffice for some people some of the time, it is not likely sufficient for most people most of the time. ${ }^{18}$ However, one might further postulate that over time, many of the needs people have for worldviews could change or be reduced, perhaps increasing the receptiveness for the kinds of cosmophilosophical/cosmological views explored here (for example, including a kind

humanism, perhaps then giving rise to a more complete worldview for some.

17 A Pew Research Center report (2012), “The Global Religious Landscape", notes that many unaffiliated people still hold religious or spiritual beliefs such as believing in God or a "higher power" (68\% in the United States)

18 If it has not already been, this question can probably be empirically addressed with psychological research. of "secular bootstrapped cosmocultural evolution" worldview). A proxy, or analog, for this suggestion is research that shows the more socially stable, comfortable, and educated people are, the less religious they apparently are (Barro and McCleary 2003, Gill and Lundsgaarde 2004), perhaps further implying less need for comprehensive worldviews that definitively address uncertainties and fears to adequately meet human needs.

As we become more knowledgeable about human emotions, and how to better deal with fear, uncertainty, fairness, and human relations more generally, we may find an increasing receptiveness to alternative worldviews that may be less specific, less prescriptive, less personal, less comprehensive, less definitive. ${ }^{19}$ As our knowledge and "caring capacity" improves, we may be able to care for each other better (Lupisella 2013) and perhaps then increasingly tolerate a variety of uncertainties. ${ }^{20}$ Decreases in religiosity in many parts of the world may be an indicator that this kind of trend is already underway. Our descendants may be better equipped to be more receptive to alternative worldviews, including cosmological worldviews.

Speculating further, forms of artificial intelligence may have very different needs regarding worldviews - including that they may not need any at all (at least in the way we think about worldviews today). Presumably, however, artificial intelligence will need

19 Van den Bos (2009) suggests that cultural worldviews are a way to cope with personal uncertainty. Van den Bos and Lind (2009) suggest that the way people assess fairness has much in common with the social psychology involved in defending worldviews. Related, Henriques (2011) suggests that humans are "the justifying animal" - uniquely powerful creators of justification systems. This seems consistent with the idea that one of the functions of worldviews is to help justify many aspects of the human condition - e.g. what we value and why, why we are here and do what we do, what our aspirations ought to be, etc.

20 A significant challenge that many intelligent beings may face is to at least tolerate, if not ultimately accept, the enduring uncertainty of an apparently objectively "pointless" universe (Lupisella 2009). 
something to guide actions, but such entities may not have the kinds of human needs we see today that are, at least in part, if not completely the result of Darwinian evolution. The more capable a species becomes, the more choices there are, the more values there can be, the more philosophy matters (Lupisella 2015). Artificial intelligence, or superintelligence more generally, will presumably be able to explore broad possibility spaces very quickly and have a high tolerance for uncertainty and indifference regarding a lack of broader "objective" meaning or purpose, or the need to be cared for, or to exist forever, etc. For our descendants, or for other advanced beings, either biological or "post-biological" (Dick 2003, Schneider 2015), the universe may indeed "be enough". ${ }^{21}$

The working hypothesis from this brief examination suggests that the universe, in the form of "cosmological worldviews" that focus on scientific cosmic evolution, are probably not enough for most people most of the time, but could be enough for some people some of the time to suffice as a basis for worldviews. However, as we evolve further, the universe may increasingly suffice - particularly as more advanced intelligence evolves. If we are not satisfied with worldviews we see today, then we can keep working on new ones. Our Cosmos seems to be a good place to start.

21 Bell (2016) explicitly treats questions regarding the relationships between superintelligences and worldviews.

\section{References}

Aerts, Diederik, and Hubert Van Belle, Jan Van der Veken. 1994. Worldviews: From Fragmentation to Integration, Brussels, VUBPRESS.

Aerts, Diederik, and Hubert Van Belle, Jan Van der Veken. 1999. World Views and the Problem of Synthesis. Springer and VUB University Press. Originally published by Kluwer Academic Publishers

Babbage, D. R. \& Ronan, K. R. 2000. Philosophical worldview and personality factors: studying the world in our own image. Personality and Individual Differences, 28(2), 405-420.

Barlow, Connie. 1997. Green Space, Green Time: The Way of Science. New York: Springer-Verlag.

Barrow, John and Tipler, Frank. 1994. The Anthropic Cosmological Principle. Oxford University Press.

Barro, Robert J. and McCleary, Rachel M. 2003. International Determinants of Religiosity. Working Paper 10147 of the National Bureau of Economic Research. http://www.nber.org/papers/w10147

Bell, David. 2016. Superintelligence and Worldviews: Putting the Spotlight on Some Important Issues. Guildford, Surrey, UK: Grosvenor House Publishing Limited.

Bohm, D. 1989. Meaning and Information, in The Search for Meaning: The New Spirit in Science and Philosophy, ed. P.Pylkkänen. Wellingborough: Crucible

Bohm, David and Hiley, Basil, J. 1984. Measurement understood through the quantum potential approach. Foundations of Physics 14, 3, 255-274.

Bohm, David and Hiley, Basil J. 1987. An Ontological Basis for Quantum Theory: I. Non-relativistic Particle Systems. Phys. Rep. 144 (6): 323-348.

Bohm, David and Basil Hiley. 1993. Undivided universe: An Ontological Interpretation of Quantum 
Theory. New York: Routledge.

Bostrom, N. 2003. Are You Living in a Simulation? Philosophical Quarterly, Vol. 53, No. 211, pp. 243-255.

Carroll, Sean. 2016. The Big Picture: On the Origins of Life, Meaning, and Universe Itself. New York: Dutton.

Christian, David. 2004. Maps of Time: an Introduction to Big History. Berkeley: University of California Press.

Crawford, Ian 2018. Big History and the Cosmic Perspective. Astronomy and Geophysics 59, 5.335.36 .

Davies, Paul. 2009. Life, Mind, and Culture as Fundamental Properties of the Universe in Cosmos and Culture: Cultural Evolution in a Cosmic Context, edited by Steven J. Dick and Mark Lupisella. Washington, DC: NASA History Series. http://history.nasa.gov/SP-4802.pdf.

Davies, P. and N. Gregersen. 2010. Information and the Nature of Reality: From Physics to Metaphysics. Cambridge University Press.

Dick, Steven. J. 2000. Cosmotheology: Theological Implications of the New Universe, in Many Worlds: The New Universe, Extraterrestrial Life, and the Theological Implications, edited by Steven J. Dick, 191-210. Philadelphia: Templeton Foundation Press.

Dick, Steven J. 2003. "Cultural evolution, the Postbiological Universe and SETI," International Journal of Astrobiology 2, no.1, 65-74.

Dick, S. 2018. Astrobiology, Discovery, and Societal Impact. Cambridge University Press.

Dick, S. and Lupisella, M. 2009. Cosmos and Culture: Cultural Evolution in a Cosmic Context. National Aeronautics and Space Administration. Online version here: https://history.nasa.gov/SP4802.pdf
Gabora, L. \& Aerts, D. 2009. A model of the emergence and evolution of integrated worldviews. Journal of Mathematical Psychology, 53, 434451.

Gardner, James. 2005. "Coevolution of the Cosmic Past and Future: The Selfish Biocosm as a Closed Timelike Curve." Complexity 10, no. 5 (May/ June 2005): 14, 17-18.

Bulbulia, J., and R. Sosis, E. Harris, R. Genet, C. Genet, K. Wyman, eds.. 2008. The Evolution of Religion: Studies, Theories, and Critiques Collins Foundation Press, Santa Margarita (CA).

Gershenson, Carol and Diedrerik Aerts, Bruce Edmonds, eds. 2007. Worldviews, Science and Us: Philosophy and Complexity. World Scientific Publishing.

Gill, Anthony and Lundsgaarde, Erik. 2004. State Welfare Spending and Religiosity: A Cross-National Analysis. Rationality and Society, Vol.16(4):399-436.

Goodenough, Ursula. 1998. The Sacred Depths of Nature. Oxford University Press.

Guth, Alan, and Paul Steinhardt. 1984. The Inflationary Universe. Scientific American, May 1984.

Guth, Alan. 1998. The Inflationary Universe: The Quest for a New Theory of Cosmic Origins. Perseus Books.

Haidt, Jonathan. 2012. The Righteous Mind: Why Good People Are Divided By Politics and Religion. New York: Random House.

Harris, Sam. 2005. The End of Faith. New York: W.W. Norton \& Company.

Hedlund-de Witt, Annick, Joop de Boer and Jan J. Boersema. 2014. Exploring worldviews and their relationships to sustainable lifestyles: Towards a new conceptual and methodological approach. Ecological Economics, Volume 84, December 2012, pp 74-83 
Henriques, Gregg. 2011. A New Unified Theory of Psychology. Springer Science+Business Media LLC.

Johnson, K. A., Hill, E. D., \& Cohen, A. B. (2011). Integrating the study of culture and religion: Toward a psychology of worldview. Social and Personality Psychology Compass, 5, 137-152

Juergensmeyer, Mark. 2003. Terror in the Mind of God: The Global Rise of Religious Tolerance. University of California Press.

Koltko-Rivera, Mark E. 2004. The Psychology of Worldviews. Review of General Psychology, Vol. 8, No. $1,3-58$

Krauss, Lawrence. 2012. A Universe From Nothing: Why is There Something Rather than Nothing? New York: Free Press

Linde, Andrei. 1994. The Self-Reproducing Inflationary Universe, Scientific American, November 1994.

Lloyd, Seth. 2006 Programming the Universe: A Quantum Computer Scientist Takes on the Cosmos. New York: Random House.

Lovejoy, Arthur. 1936. The Great Chain of Being. Harvard University Press.

Lupisella, Mark. 2009. "Cosmocultural Evolution: The Coevolution of Cosmos and Culture and the Creation of Cosmic Value." In Cosmos and Culture: Cultural Evolution in a Cosmic Context, edited by Steven J. Dick and Mark L. Lupisella, 321-359. NASA SP-2009-4802. http://history. nasa.gov/SP-4802.pdf.

Lupisella, Mark. 2013. "Caring Capacity and Cosmocultural Evolution: Potential Mechanisms for Advanced Altruism.” In D. A. Vakoch (ed.) Extraterrestrial Altruism. Berlin: Springer-Verlag.

Lupisella, Mark. 2015. Life, Intelligence, and the Pursuit of Value in Cosmic Evolution. In The
Impact of Discovering Life Beyond Earth, ed. Steven. J. Dick. Cambridge University Press.

Lupisella, Mark. 2016. Cosmological Theories of Value: Relationalism and Connectedness as Foundations for Cosmic Creativity, in The Ethics of Space Exploration, eds. James S.J. Schwartz and Tony Milligan. Space and Policy series, Springer.

Lupisella, Mark, in-press, Cosmological Theories of Value: Science, Philosophy, and Meaning in Cosmic Evolution. Springer.

Marshall, Alan. 2002. The Unity of Nature: Wholeness and Disintegration in Ecology and Science. London: Imperial College Press.

Mermin, N. David. 2017. Why QBism Is Not the Copenhagen Interpretation and What John Bell Might Have Thought of It. In Bertlmann, Reinhold; Zeilinger, Anton, eds. Quantum [Un] Speakables II. The Frontiers Collection. Springer International doi:10.1007/978-3-319-38987-5_4.

Moore, G. E. 1903. Principia Ethica. Cambridge University Press.

NASA Technical Memorandum, 1999, Workshop on the Societal Implications of Astrobiology, Final Report. November 16-17, 1999. NASA Ames Research Center

Nilsson, A. 2013. The psychology of worldviews: Toward a non-reductive science of personality (Doctoral dissertation, Lund University, Sweden). Retrieved 20181114 from http://lup.lub.lu.se/ record/3738772

Nilsson, A. 2014b. Personality psychology as the integrative study of traits and worldviews. New Ideas in Psychology, 32, 18-32.

Nilsson, A. 2015. A Q-methodological study of personal worldviews. Journal for Person-Oriented Research, 4(2), 78-94. 
Obasi, Ezemenari Marquis. 2002. Construction and Validation of the Worldview Analysis Scale. Ohio State University.

Pew Research Center, April 5, 2017, “The Changing Global Religious Landscape"

Pew Research Center report. 2012. "The Global Religious Landscape: A Report on the Size and Distribution of the World's Major Religious Groups as of 2010" http://assets.pewresearch.org/ wp-content/uploads/sites/11/2014/01/global-religion-full.pdf

Pickering, J. 1995. Active Information in Physics and in Ecological Psychology. In New Directions in Cognitive Science: Proceedings of the International Symposium, Saariselkä, Lapland, Finland, eds. P. Pylkkänen and Pylkkö. 4-9 August 1995, Helsinki: Hakapaino.

Pylkkänen, P. 2016. Quantum Theory, Active Information and the Mind-Matter Problem, in Contextuality from Quantum Physics to Psychology, eds. Ehtibar Dzhafarov, Scott Jordan, Ru Zhang, Victor Cervantes, pp 325-334. Advanced Series on Mathematical Psychology, World Scientific Publishing.

Race, M. et al. 2012. Astrobiology and Society: Building an Interdisciplinary Research Community. Astrobiology. 2012 Oct 12(10): 958-965.

Rockmore, Tom and Beth J. Singer. Antifoundationalism Old and New. 1992. Temple University Press

Rovelli, C. 1996. "Relational quantum mechanics", International Journal of Theoretical Physics, 35: 1637-1678.

Sakurai, J. J. and Jim Napolitano. 2017. Modern Quantum Mechanics, $2^{\text {nd }}$ ed. Cambridge University Press

Saucier, G. 2013. Isms dimensions: Toward a more comprehensive and integrative model of be- lief-system components. Journal of Personality and Social Psychology, 104(5), 921-939.

Saucier, G., Kenner, J., Iurino, K., Malham, P. B., Chen, Z., \& Thalmayer, A. G.., et al. 2015. Crosscultural differences in a global "survey of world views". Journal of Cross-Cultural Psychology, 46(1), 53-70.

Sciama, D. W. 1959. The Unity of the Universe. Garden City: Doubleday \& Company

Schneider, Susan. 2015. Alien Minds, in The Impact of Discovering Life Beyond Earth, ed. Steven J, Dick, pp. 189-206. Cambridge University Press.

Shermer, Michael. 2002. Why People Believe Weird Things. Henry Holt and Company.

Sloan Wilson, David. 2002. Darwin's Cathedral: Evolution, Religion, and the Nature of Society. University of Chicago Press.

Smart, John. 2009. "Evo Devo Universe? A Framework for Speculations on Cosmic Culture" in Dick, S. J. and M. Lupisella (eds.) Cosmos and Culture: Cultural Evolution in a Cosmic Context. Washington, DC: NASA History Series.

Smolin, Lee. 1997. The Life of the Cosmos. New York: Oxford University Press.

Steinhardt, P. J. and N. Turok, 2002. A Cyclic Model of the Universe. Science 296, 1436

Swimme, Brian and Thomas Berry. 1992. The Universe Story: From the Primordial Flaring Forth to the Ecozoic Era: A Celebration of the Unfolding of the Cosmos. Harper.

Tegmark, Max. 2003. "Parallel universes. Not just a staple of science fiction, other universes are a direct implication of cosmological observations". Scientific American 288 (5): 40-51. May 2003.

Teilhard de Chardin, Pierre. 1955. The Phenomenon of Man. New York: Harper Row. 
Tipler, Frank. 1994. The Physics of Immortality. New York: Doubleday.

Turchin, Valentin F. 1993. "The Cybernetic Ontology of Action." Kybernetes 22 (2): 10-30.

Van den Bos, Kees and E. Allan Lind. 2009. The Social Psychology of Fairness and the Regulation of Personal Uncertainty. In R. M. Arkin, K. C. Oleson, \& P. J. Carroll (Eds.), Handbook of the uncertain self (pp. 122-141). New York: Psychology Press.

Van den Bos, Kees. 2009. Making Sense of Life: The Existential Self Trying to Deal with Personal Uncertainty, Psychological Inquiry, 20:4, 197-217.

Vidal, Clement. 2012. "Metaphilosophical Criteria for Worldview Comparison.” Metaphilosophy 43 (3): 306-347.

Vidal, Clement. 2014. The Beginning and the End: The Meaning of Life in a Cosmological Perspective. Springer.

Von Neumann, John. 1932. The Mathematical Foundations of Quantum Mechanics. Beyer, R. T., trans., Princeton Univ. Press. 1996 edition.

Wheeler, John A. 1990. "Information, physics, quantum: The search for links", In Complexity, Entropy, and the Physics of Information, W. Zurek, ed. Redwood City: Addison-Wesley.

Whitehead, Alfred North. 1929. Process and Reality: An Essay in Cosmology. New York: Macmillan. Edition 1978 by D. R. Griffin and D. W. Sherbourne, New York: Macmillan.

Wilson, David S. 2002. Darwin's Cathedral: Evolution, Religion, and the Nature of Society. The University of Chicago Press 